\title{
Producción bibliográfica de los historiadores de El Colegio de México
}

\author{
Cristina Restrepo Arango **
}

Artículo recibido:

17 de enero de 2011.

Artículo aceptado:

28 de junio de 2011.

\section{RESUMEN}

Analiza las características demográficas de las publicaciones producidas por los investigadores del Centro de Estudios Históricos (CEH) de El Colegio de México (COLMEX) desde 1948 hasta junio del 2010. Estudia también la visibilidad y el impacto de las revistas usadas por los investigadores del CEH. Se encontraron 4,304 documentos publicados en ese periodo. Esta producción se concentran en cinco tipos de documentos: capítulos de libros $(23.95 \%)$, artículos $(22.8 \%)$, ponencias $(21.07 \%)$, libros $(14.75 \%)$ y reseñas $(11.13 \%)$. Estos documentos son publicados principalmente en

* Este artículo es parte de la tesis presentada para optar al título de Maestra en Bibliotecología en la Biblioteca Daniel Cosío Villegas de El Colegio de México. Agradezco especialmente a la Mtra. Micaela Chávez Villa y a la Dra. Lourdes Quiroa por sus observaciones para mejorar los resultados de esta investigación.

**Pontificia Universidad Javeriana, Colombia. crestrepoarango@yahoo.com

INVESTIGACIÓN BIBLIOTECOLÓGICA, Vol. 25, Núm. 54, mayo/agosto, 2011, México, ISSN: 0187-358X. pp. 111-140 
México (70.8\%) y en el idioma español (87.04\%). La revista Historia Mexicana concentra la mayor cantidad de artículos y reseñas publicadas. La revista con el índice h y JIF más alto fue Hispanic American Historical Review. La producción de documentos crece de forma exponencial.

Palabras clave: Producción científica; Centro de Estudios Históricos, El Colegio de México; Índice $\mathrm{h}$ de las revistas; Ley de Bradford; Crecimiento exponencial; Factor de Impacto de las Revistas.

\section{ABSTRACT}

Biblographical productivity of historians from El Colegio de Mexico

Cristina Restrepo Arango

The demographic characteristics of the publications produced by researchers at the Centre for Historical Studies (CEH) of El Colegio de Mexico (COLMEX) from 1948 to June 2010 are analyzed. The visibility and impact of the journals used by researchers at the CEH it is also studied. 4.304 documents published in that period were found. This production is concentrated in five types of documents, book chapters $(23.95 \%)$, papers $(22.8 \%)$, congresses' presentations $(21.07 \%)$, books $(14.75 \%)$ and critical reviews $(11.13 \%)$. These documents are published primarily in Mexico (70.8\%) and in Spanish language (87.04\%). The journal Historia Mexicana holds the largest number of articles and reviews published. The journal with the highest h-index and JIF index was Hispanic American Historical Review. The bibliographical production of these researchers is growing exponentially.

Keywords: Scientific production, Center for Historical Studies, El Colegio de Mexico; Index h of journals; Bradford Act, exponential growth; Impact Factor Journals. 


\section{INTRODUCCIÓN}

— n el mundo contemporáneo la ciencia es reconocida como conocimiento Eorganizado, donde la información necesaria para conocer su resultado es adquirida mediante la literatura publicada. Por eso, la acumulación del conocimiento tiene un significativo proceso histórico, en tanto que los descubrimientos, para ser reconocidos y homologados como ciencia, tienen que hacer públicos sus resultados. Como afirma Ziman (1986, p. 58),

sea lo que sea lo que el científico piensa o diga individualmente, sus descubrimientos no pueden ser garantizados como pertenecientes al conocimiento científico hasta que no hayan sido informados al mundo entero y puestos en un registro permanente. La institución social básica de la ciencia es así su sistema de comunicación pública.

La ciencia se caracteriza entonces por ser del conocimiento público y la literatura producida sobre un asunto científico es tan importante como la propia investigación que la incorpora, porque para la investigación científica la comunicación es esencial, ya que el objetivo final de un científico es "crear, criticar, y contribuir para alcanzar un consenso racional de las ideas y de la información de esas ideas" (Ziman, 1976, p. 318). Por tanto en la práctica científica la comunicación de las ideas se torna fundamental e involucra la publicación de artículos en revistas académicas, libros, capítulos de libros, folletos, anales de conferencias y seminarios, entre muchos otros medios de comunicación.

Estos medios de comunicación conforman el archivo histórico de la ciencia y son esenciales para mantener un registro público permanente de los resultados, observaciones, cálculos y teorías, y sirven como base para posteriores referencias de otros científicos. Ese archivo histórico de la ciencia es necesario también para

proporcionar oportunidades para la crítica, la refutación y el refinamiento de los hechos supuestos y que, por su propia naturaleza, es un cuerpo de conocimiento público al cual cada investigador hace su contribución personal (Ziman, 1986, p. 90).

Esa refutación, refinamiento y mutuo criticismo se realiza, en parte, mediante las referencias a los documentos originales. Como la comunicación científica es de interacción entre científicos, porque ellos siempre diseminan el resultado de sus investigaciones, la mayor parte de esta actividad es pública 
y rápidamente analizada por los pares (Garvey y Griffith, 1979). Luego ese trabajo de publicación y circulación de información se convierte así en un sistema de comunicación científica que le facilita a cada científico el conocimiento de todos los trabajos relevantes de otros investigadores de su campo hasta el punto de que

cuanto más eficiente es el sistema de comunicación científico, menor la existencia de duplicaciones innecesarias de investigaciones y mayor la base cultural común sobre la cual depende el avance de la ciencia (Cole y Cole, 1968, p. 397).

El esfuerzo individual de los científicos es la producción de nueva información, ya sea describiendo nuevos datos, formulando nuevos enfoques e integrando los datos conceptualmente en sus escritos. Para que esas formulaciones sean exitosas las contribuciones a la ciencia deben ser comunicadas claramente, para ser comprendidas y verificadas por otros científicos y después usadas para proporcionar nuevas bases para mayores exploraciones (Garvey y Griffith, 1979). Como ya es sabido, para hacer nuevas investigaciones los científicos se basan en investigaciones realizadas por sus predecesores y a través de la revisión de lo realizado por sus antecesores obtienen una mueva mirada a la explicación de los fenómenos, pero los resultados casi siempre son comunicados mediante la publicación formal, de tal modo que

el sistema de publicación de la ciencia es el conjunto de elementos y pautas que sostienen, regulan y perpetúan el proceso por el que los investigadores hacen accesibles de modo 'oficial' al resto de la comunidad sus pretensiones de contribuir al acervo mundial (Maltrás, 2003, p. 17).

Ese sistema de publicación de la ciencia funciona evaluando los escritos enviados por los investigadores para publicación en determinadas revistas especializadas. Esas publicaciones se convierten así en objeto de escrutinio por organismos encargados de evaluar y valorar la producción bibliográfica.

Por lo tanto una de las maneras de estudiar una disciplina académica es a través de las publicaciones pues éstas son la "expresión del estado de un autor o grupo de autores en un momento particular" (Price, 1973, p. 6). Por esa razón, la literatura producida se constituye en una especie de retrato del estado del conocimiento alcanzado y desarrollado en un determinado campo y en un determinado periodo. El análisis de esa literatura producida permite observar un campo científico y revelar sus múltiples patrones de comportamiento: autoría, tendencias de publicación, uso de la literatura producida, crecimiento de la literatura, obsolescencia de la literatura y otros. Este análisis puede 
hacerse a través de la bibliometría que permite analizar las publicaciones de un área del conocimiento. Existen trabajos bibliométricos que han analizado diversas áreas temáticas en países distintos a México como es el caso de la historia americana (Pao, 1981), la antropología americana (Rogge, 1976; Autry y Griffis, 2005), la antropología brasileña (Urbizagástegui Alvarado y Oliveira, 2001), la antropología argentina (Cajaraville, 1999), la demografía de la India (Asha, 2007), la economía americana (Sutter y Kocher, 2001), la economía de la India (Bino; Subahs y Ramanathan, 2005), la geografía (Stoddart, 1967; Aiyepeku, 1976), la música y la industria musical (Pao, 1982; Cook, 1989; Cox, Felton y Chung, 1995) y así sucesivamente en muchas otras áreas de las ciencias sociales. Sin embargo en México se carece de investigaciones similares en el campo de la historia, aunque en otras áreas de las ciencias sociales existen trabajos bibliométricos que analizan la economía mexicana como los de Puchet (2001) y Esquivel Hernández (2002), y algunas tesis de licenciatura y maestría que han estudiado las revistas de las ciencias sociales y humanidades como es el caso de la revista de Estudios Demográficos y Urbanos (Piña Pozas, 2007) y la Revista Fem (Romero Corona, 2005), así como el trabajo que analiza la revista Historia Mexicana (Cid Carmona y Ruvalcaba Burgos, 2010). Esta investigación intenta ampliar este panorama pero en el campo de la historia mexicana, debido fundamentalmente a que en el país existen esfuerzos para crear mecanismos que faciliten la difusión de las fuentes donde los investigadores hacen públicas sus investigaciones como es el caso de la creación del Portal Indicadores Bibliométricos de Clase y Periódica (BIBLAT), el Latindex y el Proyecto SCIELO-México y otros similares. Esas acciones tornan patentes la necesidad de analizar las publicaciones de los investigadores que producen conocimiento en el país. También se debe tener presente el aumento creciente de la actividad científica de El Colegio de México (COLMEX) como una institución que genera nuevos conocimientos en el país.

Esta investigación se propone identificar las características demográficas, el crecimiento de la producción bibliográfica y la visibilidad y el impacto de las revistas en las que publican sus artículos los investigadores de historia de El Colegio de México que son integrantes del Sistema Nacional de Investigadores (SNI), a través del estudio de la literatura publicada en forma de libros, artículos, ponencias presentadas en congresos, etc. Los investigadores que tienen el reconocimiento del SNI deben difundir sus investigaciones en revistas arbitradas o en libros publicados por editoriales académicas reconocidas en el ámbito científico. De la calidad de esa producción dependen tanto la aceptación de publicación de esos documentos por las revistas de la llamada "corriente principal" como la inclusión e indización de esa producción en bases de datos bibliográficas nacionales e internacionales. Estos elementos, 
si bien no garantizan su difusión, por lo menos facilita su exposición a los eventuales interesados en su uso y divulgación. Siendo el COLMEX un espacio de producción científica se espera que sus investigadores de historia produzcan conocimientos ajustados a esos criterios. Los investigadores de historia del Centro de Estudios Históricos (CEH) integrantes del SNI producen información valiosa, pero no conocemos específicamente dónde publican los resultados de sus investigaciones, ni cuál es el impacto de los documentos publicados, ni en qué idiomas los publican. Por último, no sabemos si esas publicaciones son reconocidas y visibles para la comunidad académica en ciencias sociales y humanidades en el ámbito nacional y menos para la extranjera. Por ello es importante dar respuesta a las siguientes preguntas: ¿cuáles son las características demográficas de la producción académica de esos Investigadores?, ¿cómo difunden su producción académica?, ¿qué tipo de documentos publican?, ¿dónde los publican y en qué idiomas?, ¿cuál es la tasa de crecimiento de la producción científica de estos investigadores? y ¿si las revistas a través de las cuales difunden sus publicaciones son visibles para la comunidad científica del campo?

\section{Metodología}

La población analizada en esta investigación es la producción bibliográfica publicada en forma de libros, capítulos de libros, artículos, ponencias presentadas en congresos, seminarios, coloquios, reuniones, folletos, reseñas críticas, traducciones, presentaciones y preliminares de libros (prólogos, presentaciones e índices de revistas), audiovisuales y notas publicadas en periódicos, de los investigadores del Centro de Estudios Históricos (CEH) de El Colegio de México (COLMEX) desde 1948 hasta junio del 2010. Para recolectar la producción bibliográfica publicada por esos investigadores desde los inicios de sus carreras académicas se usaron las publicaciones referenciadas en los Informes anuales del COLMEX, al igual que las referencias bibliográficas incluidas en el catálogo de la Biblioteca Daniel Cosío Villegas. También se consultaron las publicaciones listadas en el currículo de cada investigador. Todos esos datos fueron posteriormente complementados con búsquedas bibliográficas de las publicaciones de cada uno de esos investigadores indizadas en las bases de datos Academic Search Complete, Current Contents, HAPI, Humanities Abstracts, Humanities Full Text, Jstor, Social Science Full Text, Sociological Abstracts, Scopus y Web of Science. Toda esta información se manejó en el software administrador de bibliografías Pro-cite versión 5.0 conformó una base de datos bibliográfica especializada (Historia_PD). 
No se incluyeron en esta investigación comentarios o coordinaciones de mesas redondas en eventos como congresos, seminarios, coloquios, etc.; materiales en prensa o dictámenes. Tampoco se incluyeron reimpresiones, prólogos o introducciones a libros en los que los investigadores eran coordinadores, compiladores o editores.

Para analizar la producción bibliográfica de cada investigador según el tipo de documento producido en sus diferentes formas (artículos, libros, capítulos de libros, ponencias, reseñas críticas, etc.) se utilizó la estadística descriptiva. Para identificar las revistas que usan los investigadores para divulgar los resultados de sus investigaciones se usó la Ley de Bradford. Esta ley bibliométrica establece que si las revistas que contienen artículos sobre un asunto determinado fuesen ordenadas en forma decreciente de acuerdo con el número de artículos que contengan sobre ese asunto, surgen zonas sucesivas de revistas que contienen el mismo número de artículos sobre el asunto, en forma de una serie geométrica simple:

$\mathrm{n}: \mathrm{n}^{2}: \mathrm{n}^{3} \ldots$

donde,

n representa el número de revistas identificadas en la zona nuclear.

En la medida que el crecimiento de la producción bibliográfica representa el aumento de la población en una proporción fija cada año, se analizó el crecimiento de las publicaciones de los investigadores del CEH en su conjunto, ya que

cuando los valores de la variable y forman una progresión geométrica, mientras que los valores correspondientes de la variable $\mathrm{x}$ forman una progresión aritmética, la relación entre ambas variables es determinada por una función exponencial (Gupta \& Karisiddappa (2000, p. 333).

Siguiendo a Egghe y Rao (1992), esta función es matemáticamente representada como:

$$
C(t)=C(O) e^{a t}
$$

que puede ser re-escrita como:

$$
C(t)=c g^{t}
$$


donde

$\mathrm{C}(\mathrm{t})$ denota el número total de documentos producidos en el tiempo $\mathrm{t}$ g es una constante estimada de los datos observados y t es el número de $0,1,2, \ldots \mathrm{n}$ años cronológicos estudiados en esta ecuación, $\mathrm{c}>0, \mathrm{~g}>1, \mathrm{y} \mathrm{t} \geq 0$.

Para analizar la visibilidad de las publicaciones se utilizó el índice h y el factor de impacto de las revistas (JIF). El índice h consiste en tomar cada una de las revistas en las que un autor publicó sus artículos, y ordenarlos en forma descendente en función de las citas recibidas. Estos valores se ordenan en una lista de números, una ascendente (rangos) y una descendente (citas) que en un determinado punto de cruce permiten obtener el índice h. Estos datos se recuperaron a través del Journal Citation Report, Publish or Perish, Scimago Journal \& Country Rank y Web of Science. Esas bases de datos son las únicas que proporcionan las citas de forma jerarquizada tanto de libros como de los artículos publicados en las diferentes revistas y en los diferentes idiomas. De esa manera facilitan el cálculo del índice h y del JIF.

El análisis estadístico de cada uno de estos modelos bibliométricos se realizó utilizando Microsoft Excel y SPSS (versión 17.0 para Windows).

\section{RESUlTADOS Y DisCUSIONES}

La producción bibliográfica total encontrada en esta investigación fue de 4,304 documentos publicados desde 1948 hasta junio de 2010 por los investigadores que componen el personal académico del Centro de Estudios Históricos (CEH) del COLMEX incluidos en el SNI. Ese total de documentos ordenados según el lugar de publicación son presentados en la Tabla Núm. 1 (ver Anexo). El $70.8 \%$ de ese total fue publicado en México; lo que significa que solamente un $29.2 \%$ es publicado y difundido fuera de las fronteras del país. Se observa también concentración en la producción de cinco tipos de documentos: capítulos de libros $(23.95 \%)$, artículos $(22.8 \%)$, ponencias presentadas en congresos $(21.07 \%)$, libros $(14.75 \%)$ y reseñas $(11.13 \%)$. Estos cinco tipos de documentos aglutinan el $93.7 \%$ del total de los documentos producidos. El 6.3\% restante corresponde a las publicaciones en la forma de presentación, introducción o prólogos de libros o presentaciones de revistas, llamados aquí "preliminares" $(3.02 \%)$, audiovisuales $(1 \%)$, traducciones $(0.97 \%)$, notas publicadas en periódicos de circulación nacional $(0.8 \%)$ y folletos $(0.49 \%)$. La Gráfica Núm. 1 presenta el total de la producción discriminada por tipo de documentos. 


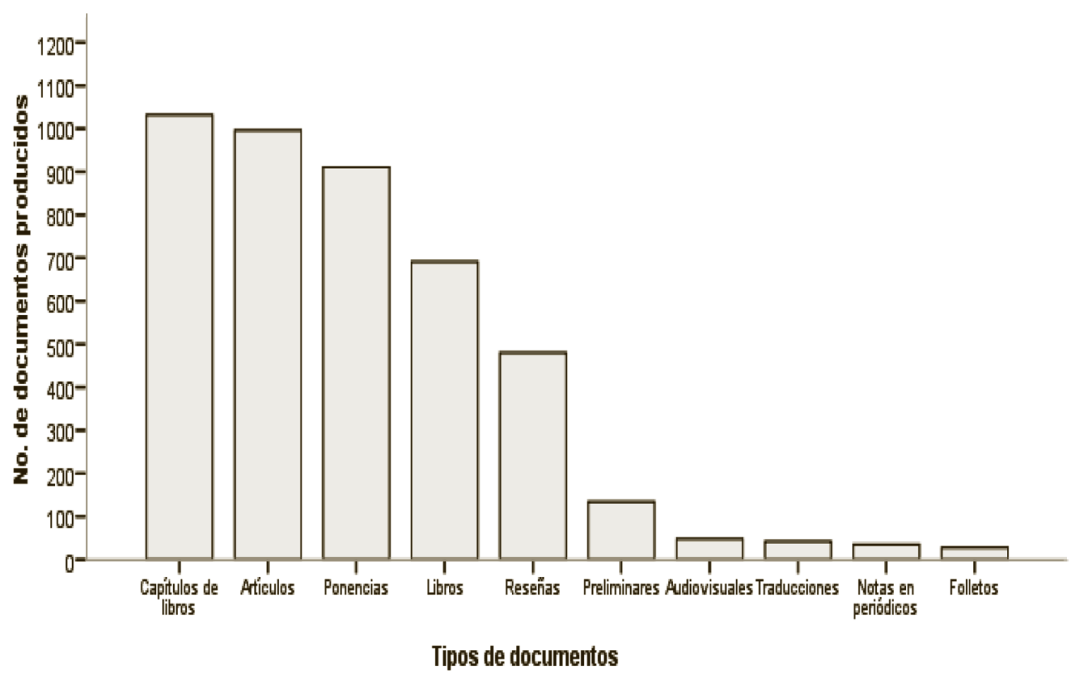

Gráfica Núm. 1: Producción científica de los investigadores por tipo de documentos

En esta gráfica se puede observar la conformación de tres grupos preferenciales de tipo de publicaciones. Con pequeñas diferencias el primer grupo está formado por capítulos de libros, artículos y ponencias presentadas en congresos o eventos similares que representan el $67 \%$ de las preferencias; un segundo grupo formado por libros y reseñas que representan el $27 \%$ de las preferencias; y el tercer grupo está conformado por preliminares, folletos, audiovisuales, notas en periódicos y traducciones con poca significación todo lo cual representa el $6.5 \%$ de las preferencias. Esta preferencia por la publicación de capítulos de libros, libros y artículos ha sido una característica de la producción historiográfica mexicana en los últimos 30 años (Serrano Álvarez, 2001). Aunque para Matute (2004, p. 328) esto se debe a que el SNI haya impuesto

modelos de evaluación provenientes de las llamadas ciencias duras [...] Por ello, será menester que se publiquen artículos en revistas de circulación internacional con arbitraje estricto. Si no fuera proclive en incurrir en lo anecdótico, señalaría que en reuniones colegiadas de evaluación del sistema se olvidó que el principal producto de los historiadores — y de los humanistas en general— es el libro. La necesidad de declarar cada tres años los avances y el temor de carecer de obras terminadas, ha favorecido el trabajo breve frente al de largo aliento, sin que se haya ideado un método confiable de evaluación de obras en proceso, de investigaciones que se desarrollan en quince o veinte años y que den por resultado obras realmente imprescindibles. 
De toda esta producción científica llama la atención la producción de reseñas que alcanzan un $11 \%$ de la producción total. En general, las reseñas dan cuenta de los principales aspectos que trata un texto con el fin de facilitar su difusión y distribución, convirtiéndose en herramientas auxiliares para la selección de publicaciones de los investigadores, bibliotecarios, libreros y especialistas en una temática (Serebnick y Cullars, 1984). También se han convertido en un mecanismo de difusión para que editores y editoriales puedan incrementar las ventas de los libros que publican. Por esta razón, se consideró pertinente analizar las revistas que seleccionan los investigadores para publicar las reseñas críticas. Se encontró que en una única revista (Historia Mexicana) se publicaron 261 reseñas (54.5\%), mientras que el $45.5 \%$ restante, es decir, 218 reseñas fueron publicadas en 80 títulos de revistas diferentes, entre las cuales destacan Hispanic American Historical Review (34 reseñas), Journal of Latin American Studies (21 reseñas) y Anglia: Anuario de Estudios Angloamericanos (10 reseñas).

En general, las reseñas se concentran en las revistas editadas en México y muy pocas en revistas foráneas (Ver la Gráfica Núm. 2); es decir, que como la difusión de las reseñas se hacen a través de la revista Historia Mexicana, parece que la preocupación es alcanzar el mercado nacional. En el COLMEX, esta revista tiene una amplia difusión debido al número de canjes con instituciones extranjeras. Quizá esto explique la concentración de reseñas en esta revista. No obstante, los editores también deberían prestar atención a la difusión de las investigaciones mexicanas en revistas de otros países de América Latina, América Anglosajona y Europa, especialmente en aquellos países con los que compartimos hechos históricos de interés común. Esa acción podría facilitar que esos textos sean conocidos, adquiridos y citados y así contribuir al impacto y visibilidad del investigador, del CEH y por ende del COLMEX en otros ámbitos académicos internacionales. 


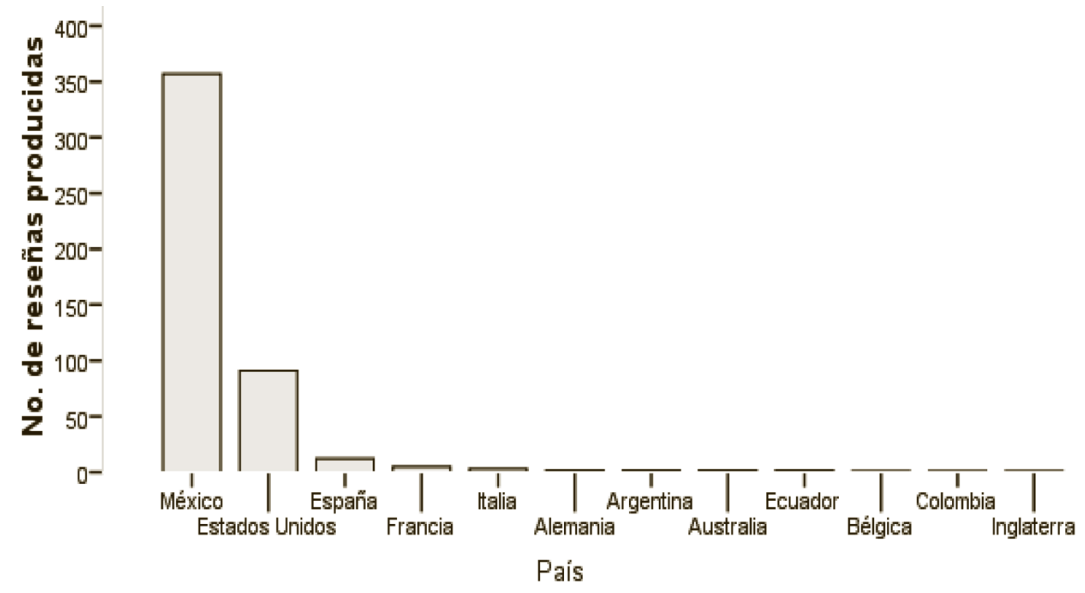

Gráfica Núm. 2: Reseñas publicadas según el país de publicación de las revistas

Otra característica demográfica importante de la producción científica es el idioma de las publicaciones. Esta demografía se presenta en la Gráfica Núm. 3. Parece natural que los investigadores del CEH prefieran publicar en el idioma oficial de México, el español, tanto que el $87.04 \%$ de la producción fue publicada en este idioma. Esto significa que hay un espacio del 12.6\% para ser compartidos con otros idiomas; el inglés es el segundo idioma en preponderancia (9\%), luego el alemán (1.32\%), el francés $(1.6 \%)$, el italiano $(1.12 \%)$ y finalmente el portugués $(0.07 \%)$. La preponderancia de publicaciones en español parece deberse también al hecho de que los pares dedicados al estudio de la historia de México necesariamente deberían leen el español, por lo que no parece se hace necesaria la publicación en otros idiomas, salvo cuando se trata de publicar en revistas de la especialidad en idiomas extranjeros. La publicación en esos idiomas extranjeros parece ser consecuencia de que los investigadores tienen como primera lengua nativa esos idiomas (inglés, italiano, alemán y francés), y en algunos casos hicieron estudios post-graduados en instituciones donde se hablan esos idiomas. 


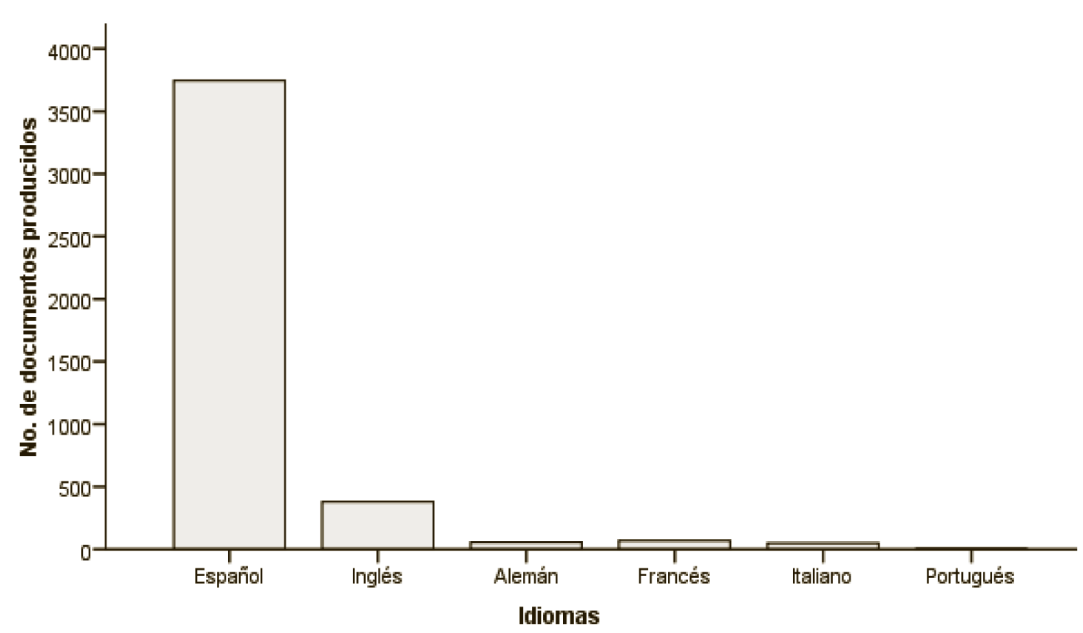

Gráfica Núm. 3: Idioma de los documentos producidos

Otra característica importante de esta producción bibliográfica es el lugar de publicación que se presenta en el Anexo 1 donde se muestra que el $70.9 \%$ de las publicaciones son editadas en México divididas entre artículos $(21.7 \%)$, libros $(16.2 \%)$, reseñas $(11.7 \%)$, capítulos de libros $(22.9 \%)$ y ponencias $(19.3 \%)$. Esta característica parece deberse al hecho de que El Colegio de México posea una editorial que facilita a los investigadores difundir sus obras, repercutiendo en la cantidad de documentos producidos en México. Existe un grupo de países (Estados Unidos, España, Francia, Italia, Austria, Alemania, Colombia, Argentina, Brasil y Reino Unido) a los que se mira con interés para la publicación de documentos, especialmente artículos y libros. Este interés parece estar relacionado con la nacionalidad de algunos de los investigadores, quienes proceden de esos países.

Las características demográficas analizadas anteriormente permitieron conocer dónde y cómo difunden los resultados de sus investigaciones los profesores del CEH, pero también es importante identificar las revistas que seleccionan prioritariamente para diseminar los resultados de sus investigaciones. Con ese objetivo, se utilizó la ley de Bradford. Las revistas se ordenaron descendentemente según el número de artículos publicados. Como se puede observar en la Tabla Núm. 1, los 981 artículos producidos fueron divididos en cuatro zonas bradfordianas como las más coherentes para medir la dispersión de los artículos publicados por los investigadores del CEH. Se encontró que el núcleo está conformado por una única revista, ésta es la revista Historia Mexicana, en la cual los investigadores han publicado un total de 239 artículos. La segunda zona está compuesta por 19 títulos de revistas 
diferentes a través de las cuales han divulgado entre 8 y 21 artículos con un total de 252 artículos. Ésta es también llamada zona de frontera, porque generalmente agrupa las revistas más cercanas al área de estudio. Se identificaron dos zonas de dispersión, la tercera zona está representada por 74 revistas y 250 artículos. La cuarta zona está representada por 215 revistas y 240 artículos. Las zonas 1 y 2 concentran el $6.5 \%$ de las revistas seleccionadas para divulgación, en las cuales se han diseminado el 50\% de las investigaciones. Para divulgar el otro $50 \%$ de las publicaciones los investigadores seleccionaron el $93.5 \%$ del total de las revistas restantes.

Tabla Núm. 1. División zonal de Bradford

\begin{tabular}{|c|c|c|c|}
\hline Zona & Núm. de revistas & Núm. de artículos & Multiplicador de Bradford \\
\hline 1 & 1 & 239 & -- \\
\hline 2 & 19 & 252 & 19 \\
\hline 3 & 74 & 250 & 3.89 \\
\hline 4 & 215 & 240 & 2.90 \\
\hline Total & 309 & 981 & 8.3 \\
\hline
\end{tabular}

Las revistas escogidas por estos investigadores son las 20 que se indican en la Tabla Núm. 2, la cual presenta los títulos de revistas, el número de artículos publicados en cada una de ellas y el lugar de publicación de los 20 títulos correspondientes al núcleo y a la zona de frontera identificados a través de la aplicación de la ley de Bradford, de ellos 15 títulos son editados en México, 2 en Estados Unidos y 1 revista en cada uno de los siguientes países: Austria, España y Francia.

Tabla Núm. 2: Principales revistas donde publican artículos los investigadores del CEH/Colmex

\begin{tabular}{|l|c|c|}
\hline \multicolumn{1}{|c|}{ Títulos de las revistas } & Núm. de artículos & Lugar de publicación \\
\hline 1. Historia mexicana & 239 & México \\
\hline $\begin{array}{l}\text { 2. Relaciones: estudios de historia } \\
\text { y sociedad }\end{array}$ & 21 & México \\
\hline $\begin{array}{l}\text { 3. Revista de la Universidad de } \\
\text { México (UNAM) }\end{array}$ & 19 & México \\
\hline $\begin{array}{l}\text { 4. Boletín editorial de El Colegio de } \\
\text { México }\end{array}$ & 19 & USA \\
\hline $\begin{array}{l}\text { 5. Mexican Studies/Estudios } \\
\text { Mexicanos }\end{array}$ & 17 & México \\
\hline $\begin{array}{l}\text { 6. } \text { Diálogos: artes, letras, ciencias } \\
\text { humanas }\end{array}$ & 17 & México \\
\hline 7. Memorias de la Academia \\
Mexicana de Historia
\end{tabular}




\begin{tabular}{|l|c|c|}
\hline $\begin{array}{l}\text { 10. Hispanic american historical } \\
\text { review }\end{array}$ & 14 & USA \\
\hline 11. Arqueología mexicana & 13 & México \\
\hline 12. Letras libres & 12 & México \\
\hline 13. Estudios sociológicos & 12 & México \\
\hline 14. Historia y grafía & 11 & México \\
\hline 15. Revista la palabra y el hombre & 10 & Austria \\
\hline $\begin{array}{l}\text { 16. Jahrbuch für geschichte } \\
\text { lateinamenkas }\end{array}$ & 9 & México \\
\hline 17. Historias & 9 & España \\
\hline 18. Revista de indias & 8 & Francia \\
\hline 19. Cahiers des Amériques Latines & 8 & México \\
\hline $\begin{array}{l}\text { 20. Boletín Archivo General de la } \\
\text { Nación }\end{array}$ & 8 & \\
\hline
\end{tabular}

Estudiando la temática de estas revistas se ha observado que no todas son del área de historia, pero sí de las ciencias sociales y humanidades. La tendencia de los investigadores a publicar en revistas del campo de las ciencias sociales y humanidades sugiere que "la historia es un fuerte componente" de esta área. Según Miño Grijalva (1991, p. 29), la historia "es patrimonio y base de todas las ciencias sociales". Asimismo, se debe tener presente que en México las revistas dedicadas al campo de la historia comienzan a especializarse en la década de los ochenta; en esa década aparecieron nuevos títulos de revistas y las ya existentes se consolidaron en el mercado y en el ámbito latinoamericano, lo cual permitió que "las revistas dedicadas a la historia [comenzaran] a separarse de las ciencias sociales y de la antropología” (Miño Grijalva y Rabadán Figueroa, 2004, p. 413), en las que se publicaban antes de esa década, y en las que ganaron, además, su propio espacio y especialización. Esas observaciones y afirmaciones se ven reflejadas en las revistas escogidas para divulgar las investigaciones del CEH, sólo 8 de estas revistas están especializadas en el campo de la historia y los 12 títulos restantes pertenecen al campo de las ciencias sociales y humanidades. También las revistas de dispersión (zonas 3 y 4) pertenecen a diferentes áreas que no son del campo de las ciencias sociales y humanidades. Esta dispersión al parecer se debe también a que

la gente de casa prefirió escribir en otros periódicos: en Diálogos, la revista de alta divulgación adoptada por El Colegio; en los órganos de la UNAM, y en no pocas ocasiones, en revistas que satisfacían la vanidad de verse impresos en inglés, francés, alemán o italiano (González, 1976, p. 567).

EL Anexo 2 relaciona los títulos de las principales revistas usadas para diseminar los resultados de las investigaciones y las bases de datos en las cuales 
han sido indizadas. Las bases de datos Hispanic American Periodicals Index (HAPI) e Historical Abstracts indizan la mayoría de estas revistas seguida de MLA. Sin embargo, sólo 4 revistas son indizadas en el Web of Science y Scopus (Historia mexicana, Mexican studies/Estudios mexicanos, Hispanic american historical review y Revista de indias). Ambas bases de datos son las que promueven la visibilidad de los investigadores y sus publicaciones a nivel internacional. Tres de las 20 revistas escogidas para difundir las investigaciones no son indizadas por ninguna base de datos que represente el campo, tanto en el ámbito nacional como en el internacional. Dos de estos títulos son boletines que sirven como medios de difusión de noticias, reseñas de libros y artículos de interés general. Para la revista Memorias de la Academia Mexicana de Historia no se encontró ninguna explicación. Estos tres títulos tampoco son indizados en CLASE y PERIÓDICA, como sí es el caso de los demás títulos de las revistas editadas en México. Llama la atención que de los 20 títulos utilizados para la difusión de los documentos publicados por estos investigadores sólo 5 revistas (Historia Mexicana, Relaciones: Estudios de Historia y Sociedad, Secuencia: Revista Americana de Ciencias Sociales, Estudios Sociológicos y Historia y Grafía) aparecen en el índice de revistas de Calidad del CONACyT (Consejo Nacional de Ciencia y Tecnología, 2010).

Esta tendencia de indización de las revistas en bases de datos nacionales es similar al caso de la producción bibliográfica española en ciencias humanas, ya que el $88 \%$ de los artículos se recogen en bases de datos producidas en ese país (Bordons, Sancho, Morillo y Gómez, 2010). Esta situación es también similar al problema que enfrenta la antropología argentina, que a pesar de contar con 4 bases de datos internacionales que supuestamente deberían recoger la producción a nivel mundial no indizan revistas latinoamericanas (Romanos de Tiratel y Giunti, 2007). También se ha observado este fenómeno en la investigación española en ciencias sociales y humanidades, donde la mayoría de los resultados de las investigaciones son difundidas en revistas no homologadas internacionalmente (Etxebarria y Gómez-Urunga, 2010). Esta exclusión se debe a que las bases de datos de dominación anglosajona no indizan la producción latinoamericana. Aunque existen bases de datos como HAPI y Handbook of Latin American Studies (HLAS) que recogen la producción de esta región y la ponen a disposición de los usuarios en diversos formatos, esas bases de datos son principalmente producidas en Estados Unidos y responden a los intereses de los usuarios de ese país. Estas bases de datos tienen limitaciones en cuanto al número de títulos que indizan, a los criterios definidos para el efecto, entre los que se destaca que las publicaciones se editen de manera regular y salgan a tiempo, lo cual no siempre cumplen las revistas latinoamericanas. En México se han hecho esfuerzos encaminados a 
indizar la producción nacional y regional a través de CLASE, PERIÓDICA, LATINDEX, HUMANINDEX y RedALyC (Rodríguez Gallardo, 2010). Sin embargo, no se debe olvidar que la mayoría de las publicaciones editadas en América Latina se caracterizan por una distribución inadecuada, lo que permite que las investigaciones más "valiosas caigan en la amplia categoría de 'ciencia pérdida', cuyos resultados en la práctica no llegan a alimentar los logros y discusiones académicas" (Castro Gutiérrez, 2010, p. 2).

También se analizó el factor de impacto (JIF) de las revistas y el índice h para estudiar la visibilidad y el impacto de los 20 títulos de las revistas más utilizadas por estos investigadores. Estos datos se recuperaron a través del Journal Citation Report, Publish or Perish, Scimago Journal \& Country Rank y Web of Science. Esas bases de datos son las únicas que proporcionan las citas de forma jerarquizada tanto de libros como de artículos publicados en las diferentes revistas y en los diferentes idiomas. De esa manera facilitan el cálculo del índice h y del JIF. Los resultados obtenidos se presentan en la Tabla Núm. 6.

En Publish or Perish (PoP), la revista Hispanic American Historical Review tiene un índice h de 38, le siguen Historia Mexicana con un índice de 22, Estudios Sociológicos con un índice de 18, Mexican Studies/Estudios Mexicanos con un índice de 15 y la Revista de Indias con un índice de 13, luego Nexos con un índice de 10, Letras libres con un índice de 8, Arqueología Mexicana con 7 e Historia y Grafía con 6. Se destaca que los valores del índice h que se obtuvieron a través de esta herramienta coinciden con que estas 5 revistas (Hispanic American Historical Review, Historia Mexicana, Estudios Sociológicos, Mexican Studies/Estudios Mexicanos y Revista de Indias) son indizadas en el Web of Science y/o Scopus. Los 10 títulos de revistas restantes tuvieron índices entre 1 y 5 . Es decir, muestran un bajo índice de visibilidad.

Tabla Núm. 3: Índice h y JIF de las revistas

\begin{tabular}{|l|c|c|c|c|}
\hline \multicolumn{1}{|c|}{ Título de la revista } & $\begin{array}{c}\text { Índice } \mathrm{h} \\
\text { PoP }\end{array}$ & $\begin{array}{c}\text { Índice } \mathrm{h} \\
\text { SJ\&CR }\end{array}$ & $\begin{array}{c}\text { Índice } \mathrm{h} \\
\text { WoS }\end{array}$ & $\begin{array}{c}\text { JIF } \\
\text { WoS }\end{array}$ \\
\hline 1. Historia mexicana & 22 & 1 & 8 & 0.00 \\
\hline $\begin{array}{l}\text { 2. Relaciones: estudios de } \\
\text { historia y sociedad }\end{array}$ & 5 & -- & -- & -- \\
\hline $\begin{array}{l}\text { 3. Revista de la Universidad de } \\
\text { México (UNAM) }\end{array}$ & 1 & -- & -- & -- \\
\hline $\begin{array}{l}\text { 4. } \text { Boletín editorial de El Colegio } \\
\text { de México }\end{array}$ & 2 & -- & 6 & 0.06 \\
\hline $\begin{array}{l}\text { 5. Mexican Studies/Estudios } \\
\text { Mexicanos }\end{array}$ & 15 & 4 & -- & - \\
\hline $\begin{array}{l}\text { 6. Diálogos: artes, letras, } \\
\text { ciencias humanas }\end{array}$ & 2 & - & & \\
\hline
\end{tabular}




\begin{tabular}{|c|c|c|c|c|}
\hline $\begin{array}{l}\text { 7. Memorias de la Academia } \\
\text { Mexicana de Historia }\end{array}$ & 4 & -- & -- & -- \\
\hline 8. Nexos & 10 & -- & -- & -- \\
\hline $\begin{array}{l}\text { 9. Secuencia: revista americana } \\
\text { de ciencias sociales }\end{array}$ & 3 & -- & -- & -- \\
\hline $\begin{array}{l}\text { 10. Hispanic american historical } \\
\text { review }\end{array}$ & 38 & 4 & 16 & 2.114 \\
\hline 11. Arqueología mexicana & 7 & -- & -- & -- \\
\hline 12. Letras libres & 8 & -- & -- & -- \\
\hline 13. Estudios sociológicos & 18 & -- & -- & -- \\
\hline 14. Historia y grafía & 6 & -- & -- & -- \\
\hline 15. Revista la palabra y el hombre & 3 & -- & -- & -- \\
\hline $\begin{array}{l}\text { 16. Jahrbuch für geschichte } \\
\text { lateinamenkas }\end{array}$ & 1 & -- & -- & -- \\
\hline 17. Historias & 4 & -- & -- & -- \\
\hline 18. Revista de indias & 13 & 2 & 4 & 0.05 \\
\hline $\begin{array}{l}\text { 19. Cahiers des Amériques } \\
\text { Latines }\end{array}$ & 4 & -- & -- & -- \\
\hline $\begin{array}{l}\text { 20. Boletín Archivo General de } \\
\text { la Nación }\end{array}$ & 5 & -- & -- & \\
\hline
\end{tabular}

En Scimago Journal \& Country Rank (SJ\&CR), las revistas Hispanic American Historical Review y Mexican Studies/Estudios Mexicanos tienen un índice de 4. La Revista de Indias tiene un índice de 2 e Historia Mexicana tiene un índice de 1. En esta herramienta sorprendentemente, las revistas muestran un bajo índice h. Este bajo índice puede deberse a que SJ\&CR solamente analiza los datos que extrae de SCOPUS. Es bien conocido el hecho de que las revistas publicadas en inglés tienen mayor visibilidad que las revistas publicadas en otros idiomas, porque son preferentemente indizadas en esas bases de datos y SCOPUS no es una excepción. Por ejemplo, en este caso, las dos revistas que publican la mayoría de sus artículos en inglés y son editados en los Estados Unidos tienen un índice de 4, mientras que las otras dos revistas que se publican en español y en países que apenas comienzan a fortalecer la investigación, tienen un índice de 1 y 2.

En Web of Science (WoS), de las 20 revistas analizadas apenas 4 muestran el índice h, por lo tanto 16 revistas ni siquiera muestran este índice, es decir, son invisibles. La revista Hispanic American Historical Review tiene un índice de 16, Historia Mexicana tiene un índice de 8, Mexican Studies/Estudios Mexicanos un índice de 6 y Revista de Indias un índice de 4. Uno de los problemas más serios del WoS es su sesgo en relación a la cobertura en favor de las revistas publicadas en inglés en detrimento de las revistas procedentes de países no anglosajones. En este caso se observa ese sesgo, puesto que de 
las 16 revistas publicadas en español solamente 2 (Historia Mexicana y Revista de Indias) muestran un índice h; es decir, la mayoría de ellas (14 revistas) son invisibles para la comunidad de investigadores del área de historia. Se ha dicho que "esto es un problema muy importante para la ciencia escrita en lengua castellana, dado que la mayoría (aproximadamente cerca del 90\%) de las revistas incluidas en el Web of Science del ISI están editadas en lengua inglesa, mientras que las revistas editadas en castellano no superan el $8 \%$ " (Buela-Casal, 2010, p. 4).

En relación con el factor de impacto (JIF), éste se recuperó a través del Journal Citation Report y Art \& Humanities Citation Index. El JIF más alto lo obtuvo la revista Hispanic American Historical Review con 2.114, le siguen Mexican Studies/Estudios Mexicanos con 0.06, Revista de Indias con 0.05.

Resumiendo, la revista Hispanic American Historical Review es la que muestra un mayor índice h y JIF en las 4 herramientas utilizadas, seguida por la revista Mexican Studies/Estudios Mexicanos. Ambas revistas son editadas en inglés y esa puede ser la razón que explique el por qué de sus mayores índices. Las revistas publicadas en español, no muestran índices elevados o ni siquiera muestran esos índices en las herramientas del WoS, porque no son indizadas. Los valores del índice h obtenidos a través del Publish or Perish son mayores, porque en esta aplicación los sesgos idiomáticos, geográficos y el tipo de documentos presentes en el Web of Science y Scopus son menores y tratan de ser evitados. Sin embargo no se puede afirmar de forma concluyente que los valores del índice h o del JIF representen una mayor o menor visibilidad o mayor o menor impacto de las revistas, más aún cuando estos valores dependen de factores extrínsecos e intrínsecos al contexto en que se edita cada revista. Para Dolfsma y Leydesdorff (2008), los valores del JIF y el índice $h$ dependen de las características individuales de cada revista, así como del área en la que cada revista se especializa. Estos autores sostienen que "las revistas en ciencias naturales tienden a tener un JIF más alto que las revistas en las ciencias sociales" (2008, p. 215). Los resultados que obtiene cada revista con estos indicadores también están asociados con los patrones de citación; el tipo de artículos que publican y las políticas editoriales adoptadas en la edición y aceptación de trabajos (Dolfsma y Leydesdorff, 2008; Haddow, 2008). Éste parece ser el caso de las revistas analizadas en este trabajo.

Otro aspecto que se estudió en este trabajo fue el crecimiento de la producción bibliográfica total desde 1948 hasta junio del 2010, lo que se representa en la Gráfica Núm. 5. Aunque la producción crece con algunas fluctuaciones ésta permaneció más o menos estable hasta 1970. A partir de esta década la producción crece hasta alcanzar su punto cúspide en el 2001. Este aumento de la productividad parece estar asociado al hecho "que los estudios 
de historia entraban en un periodo de consolidación, a nivel de un nuevo tipo de preocupaciones y problemas por investigar [...]” (Miño Grijalva, 1991, p. 26). También ese crecimiento ya había sido previsto pues de

[...] un tiempo a esta parte, se advierte un aumento considerable en la producción especializada [...] y, si bien es cierto que queda mucho por explorar, hay motivos para suponer que el volumen y la variedad de los estudios crecerán rápidamente en el futuro (Potash, 1961, p.361).

\section{Asimismo este crecimiento parece deberse a la}

expansión de la historiografía regional, [..] a la creación de centros de enseñanza o investigación, [y a que] se fundaron licenciaturas y postgrados en las universidades estatales y los gobiernos apoyaron proyectos de investigación acerca de las historias regionales de los estados. [También] los archivos gubernamentales de los estados o los municipios recibieron apoyos considerables para permitir la organización y la apertura de la investigación [histórica] (Serrano Álvarez, 2001, p.118).

Igualmente la creación del Sistema Nacional de Investigadores (SNI) parece haber impulsado la producción académica, tanto que

en los últimos 20 años ha crecido y se ha fortalecido la profesionalización de los estudios históricos en México, de manera que se ha convertido en una carrera respetada que ha visto incrementar tanto el número de practicantes como el de instituciones dedicadas a formar nuevos recursos humanos e incrementar el conocimiento histórico del país (Matute, 2004, p. 329).

Otro hecho que ha impulsado la producción Bibliográfica han sido "los premios que ofrecen instituciones académicas, las cuales otorgan estímulos económicos y la publicación de trabajos inéditos" (Matute, 2004, p. 333). Naturalmente esta amalgama de factores contribuyó al crecimiento de la producción Bibliográfica de los historiadores hasta el 2001, aunque hubo una caída notoria en 1993 y un ascenso en 1994. 


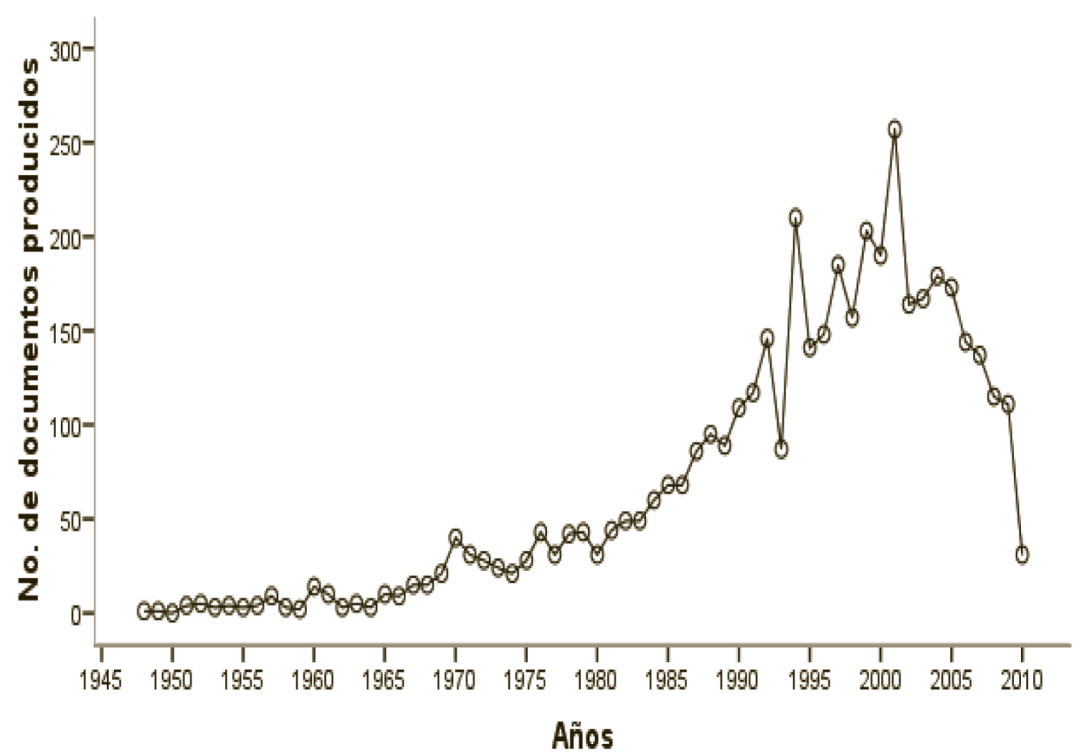

Gráfica Núm. 5: Crecimiento de las publicaciones producidas por año

A partir de 2001 se observa una caída en el crecimiento de las publicaciones que dura hasta el 2010. Matute (2004) sostiene que esta caída de la productividad es consecuencia de la disminución en la contratación de profesores, debido a las restricciones impuestas en el gasto público que no favorecen la investigación ni la enseñanza superior; de tal manera que

todas nuestras publicaciones sufren, en grados variables, las dificultades económicas propias de cualquier proyecto editorial de carácter no comercial, y las carencias por no decir vicios, de los sistemas de distribución (Alberro, 2001, p. 647).

En general, para la producción total de los investigadores del CEH se estimó una media de 69.11 documentos producidos por año con una mediana de 42.5, una moda de 3, una desviación estándar de 68.622, una varianza de 4708.954 y con un error típico de la media de 8.715 .

La representación del crecimiento de la producción total acumulada se puede observar en la Gráfica Núm. 6. Éste trazado muestra una forma cóncava al inicio de la distribución que asciende lentamente hasta alcanzar su punto máximo en el 2010. 


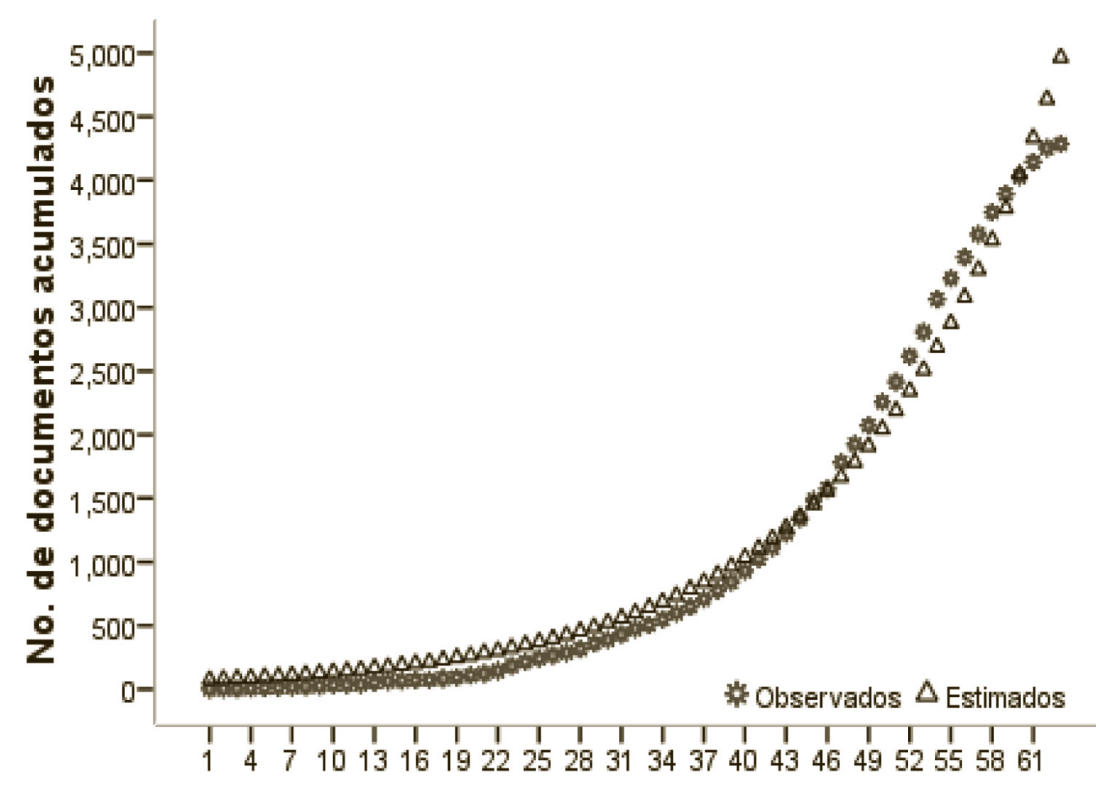

Años

Gráfica Núm. 6: Crecimiento exponencial de las publicaciones producidas por año

En general la función es creciente y la nube de puntos configura un crecimiento de forma exponencial. El R2 ajustado estimado fue igual a 0.982, indicando un buen ajuste de los datos al modelo exponencial. El valor estimado de c fue de 67.549 incluyendo un error padrón de 6.995. El valor estimado de $\mathrm{g}$ fue igual a 1.071 e incluyó un error padrón de 0.002. Una vez conocidos esos valores se puede construir la ecuación siguiente que predice el crecimiento exponencial de la producción de documentos de los investigadores del CEH:

$$
C(t)=67.549 \times 1.071^{t}
$$

Esa ecuación indica que la producción de documentos crece en una proporción media de $7.1 \%$ al año y se duplica cada 10.1 años; es decir, se duplica 10 veces en un siglo. Esta tasa de crecimiento es similar en la ciencia donde "[...] a juzgar por el número de documentos publicados en un año ... [ésta] se duplica de forma exponencial cada diez años" (Brookes, 1973, p. 500). Otros autores como Urbizagástegui Alvarado (2008), destacan que la literatura ya sea en las ciencias puras o ciencias sociales y humanidades crece exponencialmente. 


\section{Conclusiones}

Se encontró que la producción Bibliográfica publicada por los investigadores del CEH está centrada en tres grupos diferentes de tipos de publicaciones. Un primer grupo conformado por capítulos de libros, artículos y ponencias. Un segundo grupo formado por libros y reseñas, y por último un tercer grupo de preliminares, audiovisuales, folletos y traducciones. Estos trabajos son publicados en su mayoría en idioma español, pero en menor proporción seguido por el inglés, alemán, francés, italiano y portugués, y es México el lugar de publicación predominante, seguido de los Estados Unidos, España, Francia, Italia, Austria, Colombia, Argentina, Brasil, Inglaterra y Puerto Rico. Los artículos son diseminados básicamente a través de la revista Historia Mexicana complementada por otras 19 revistas, a través de las cuales se divulga el $50 \%$ de la producción de los investigadores del CEH. Hasta junio de 2010 los investigadores del CEH habían producido y divulgado 4,304 documentos. Estos documentos crecen de forma exponencial a una proporción media de $7.1 \%$ al año, y se duplica cada 10.1 años, es decir, crecen lentamente.

Aunque los artículos publicados en revistas son el segundo tipo de documentos producidos por los investigadores del CEH, esta producción de artículos no está totalmente indizada en las bases de datos revisadas y sólo 4 de las 20 revistas más utilizadas para difundir la producción son indizadas por las bases de datos Web of Science y Scopus. Además estas bases de datos no indizan los tipos de documentos que prefieren publicar los investigadores del CEH; es decir, libros, capítulos de libros y ponencias presentadas en congresos y eventos similares. Igualmente estas bases de datos no cubren la producción bibliográfica desde 1948, fecha en la que comenzaron a aparecer los primeros trabajos de estos investigadores. La cobertura temporal de las citas en Scopus se inicia en el 1996, mientras que la producción de los investigadores del CEH se inició 48 años antes. Es decir, hay una brecha diferencial temporal de exactamente 48 años que debe ser cubierta por alguna base de datos ya sea nacional o internacional que se proponga o quiera difundir la producción bibliográfica de los historiadores del CEH.

La mayoría de estas revistas no están indizadas en las bases de datos más usadas en las ciencias sociales como son el Social Science Citation Index, Art \& Humanities Index, Web of Science y Scopus. Algunas de estas revistas presentan problemas de accesibilidad, en tanto que no se encuentran disponibles en texto completo en Internet. Aunque esto no es un obstáculo para que los investigadores interesados citen los artículos de estas revistas, sería recomendable que los editores pongan a disposición pública las revistas en texto completo en el Internet, especialmente si éstas son financiadas con recursos 
públicos. Por ejemplo, en el caso de la revista Historia Mexicana ésta puede consultarse en texto completo a través de la red, pero sus artículos están protegidos y no permiten la impresión de los textos. Sería recomendable que los editores consideren la posibilidad de eliminar esta protección, más aún cuando de lo que se trata es de mejorar la accesibilidad de las revistas para que otros investigadores puedan consultarlas y por ende citarlas. Aunque la revista es accesible a través de JSTOR esta base de datos no ofrece el texto completo de los dos últimos años.

\section{BiBLIOGRAFÍA}

Aiyepeku, W. O, "The productivity of geographical authors: A case study from Nigeria”, en Journal of documentation, núm. 32, 1976, pp. 105-117.

Alberro, Solange, "El primer medio siglo de Historia Mexicana", en Historia mexicana, vol. 50, núm. 4, 2001, pp. 643-653.

Asha, B., "Bibliometric properties of demography India", en Annals of library and information studies, vol. 54, núm. 2, 2007, pp. 73-80.

Autry, C. W. y S. E. Griffis, "A social anthropology of logistics research: exploring productivity and collaboration in an emerging science", en Transportation journal, vol. 44, núm.4, 2005, pp. 2743.

Bino, Paul G. D., S. Subash y A. Ramanathan, "Inequality in knowledge activity: a case of economics journals", en European journal of comparative economics, vol.2, núm.2, 2005.

Bordons, María, Sancho, Rosa, Morillo, Fernando y Gómez, Isabel, "Perfil de actividad científica de las universidades españolas en cuatro áreas temáticas: un enfoque multifactorial”, en Revista española de documentación cientifica, vol. 33, núm. 1, 2010, pp. 9-33.

Brookes, B. C., "Numerical methodos of bibliometrics of bibliographic analysis”. En Library trends, vol. 22, núm. 1, 1973, pp. 1843, (DE, 18 de julio, 2010: http://www.ideals.illinois.edu/bitstream/handle/2142/6715/librarytrendsv22i1d_opt. pdf? sequence $=1$ ).

Buela-Casal, Gualberto, "Scientific Journal impact indexes and indicators for measuring researchers' performence", en Revista de Psicodidáctica, vol. 15, núm. 1, 2010, pp. 3-19.

Cajaraville, María Cristina, "Acerca de la producción científica en Antropología Social: artículos y autorías en publicaciones periódicas argentinas, 1983-95”, en Referencias, vol. 4, núm. 2, 1999, pp. 55-62.

Castro Gutiérrez, Felipe. "La edición de revistas digitales de historia en México: promesas, riesgos y perspectivas”, en Nuevo mundo mundos nuevos, 2010, (de, 8 de julio, 2010: http://nuevomundorevues.org/59526). 
Cid Carmona, Víctor Julián y Eduardo Ruvalcaba Burgos, "Análisis y evaluación de la revista Historia Mexicana, 1999-2008” [Presentación en Power Point], en Coloquio Internacional: Escribir y leer: Lengua, autoridad y plataforma tecnológica en revistas de historia, 24 y 25 de febrero de 2010, México, D.F., COLMEX, 2010.

Cole, Stepeh y Jonathan R. Cole, "Visibility and the structural bases of awareness of scientific research", en American sociological review, vol. 33, núm. 3, 1968, pp. 397-413.

Consejo Nacional de Ciencia y Tecnología, Índice de Revistas, México, D.F., CONACYT, 2010. (De, 15 de noviembre, 2010: http://www.conacyt.mx/Indice/Paginas/Indice4.aspx).

Cook, Kevin L., "Laws of scattering applied to popular music", en Journal of the American Society for Information Science, vol. 40, núm. 4, 1989, pp. 277-283.

Cox, Raymond A. K.; James M. Felton y Kee H. Chung, "The concentration of commercial success in popular music: an analysis of the distribution of gold records", en Journal of cultural economics, vol. 19, núm. 4, 1995, pp. 333-340.

Dolfsma, Wilfred y Loet Leydesdorff, "Journal as constituents of scientific discourse: economic heterodoxy", en On the borizont, vol.16, núm. 4, 2008, pp. 214-225.

Egghe, L. y I. K. Ravichandra Rao, "Classification of growth model based on growth and its application”, en Scientometrics, vol. 25, núm. 1, 1992, pp. 5-46.

Esquivel Hernández, Gerardo, "Producción científica e impacto de los economistas académicos en México", en Economía mexicana, vol. 11, núm.1, 2002, pp. 5-30.

Etxebarria, Goio y Mikel Gómez-Urunga, "Use of scopues and Google Scholar to measure social sciences production in four major Spanish universities”, en Scentometrics, núm. 82, 2010, pp. 333 349.

Garvey, William D. y Griffith, Belver C., "Scientific communication as a social system," en William D. Garvey, Communication: the essence of science: facilitating information exchange among librarians, scientists, engineers and students, Oxford: Pergamon, 1979, pp. 148-164.

Gupta, B.M. y C. R. Karisiddappa, "Modeling the growth of literature in the area of theoretical populations genetics", en Scientometrics, vol. 49, núm. 2, 2000, pp. 321-355.

González, Luis. "La pasión del nido", en Historia mexicana, vol. 25, núm. 4, 1976, pp. 530-598.

Haddow, Gaby, "Quality australian journals in the humanities and social sciences", en Australian Academic \& Research Libraries, vol. 39, núm. 2, 2008, pp. 79-91.

Maltrás Barba, Bruno, Los indicadores bibliométricos: fundamentos y aplicación al análisis de la ciencia, Gijón: Ediciones Trea, 2003, 287p. 
Matute, Álvaro, “La historia en México (1984-2004)", en Historia mexicana, vol. 20, núm. 2, 2004, pp. 327-342.

Miño Grijalva, Manuel y Macrina Rabadán Figueroa, "Mexican Studies / Estudios Mexicanos 20 años de historia", en Mexican Studies / Estudios Mexicanos, vol. 20, núm. 2, 2004, pp. 411-453.

Miño Grijalva, Manuel, "Historia Mexicana: historiografía y conocimiento", en Historia mexicana, vol. 41, núm. 1, 1991, pp.23-47.

Pao, Miranda Lee, "Collaboration in Computational musicology", en Journal of the American Society for Information Science, vol. 31, núm. 1, 1982, pp. 38-43.

"Bibliometric analysis of American history data by Famulus”, en ACM SIGSOC Bulletin, vol. 12-13, núm. 4-1, 1981, pp.58-59.

Piña Pozas, Ma. Maricela, Análisis bibliométrico de la revista Estudios Demográficos y Urbanos de El Colegio de México, (Tesis para optar al título de Maestría), México, D.F. El Colegio de México, Biblioteca Daniel Cosío Villegas, 2007, 128h.

Price, Derek J. de Solla, Hacia una ciencia de la ciencia, estudio preliminar y traducción de José María López Piñero, Barcelona: Ariel, 1973, 181p.

Potash, Robert A., "Historiografía del México independiente", en Historia americana, vol. 10, núm. 3, 1961, pp. 361-412.

Puchet, Martín, "Presencia en revistas académicas de los artículos sobre la economía mexicana y productividad de los economistas académicos de México", en Economía mexicana, vol. 10, núm.1, 2001, pp. 5-35.

Rogge, A. E., "A look at academic Anthropology: through a graph darkly", en American antbropologist, vol. 78, núm. 4, 1976, pp. 829-843.

Romanos de Tiratel, Susana y Graciela M. Giunti, "Las revistas argentinas de Ciencias Antropológicas: visibilidad en bases de datos internacionales", en Information research, vol. 12, núm. 4, 2007, (De; 18 de agosto, 2010: http://informationr.net/ir/12-4/paper325.html).

Romero Corona, Sonia, Seis años de la Revista Fem vistos a través de la bibliometría, (Tesis para optar al título de licenciada), Distrito Federal, México, Universidad Nacional Autónoma de México, Colegio de Bibliotecarios, 2005, 80p.

Serrano Álvarez, Pablo, “ Interpretaciones de la historiografía regional y local mexicana, 1969-1999: los retos teóricos, metodológicos y líneas de investigación”, en Revista de historia regional, vol. 6, núm. 2, 2001, pp. 113-125.

Serebnick, Judith y John Cullars, "An analysis of reviews and library holdings of small publishers books", en Library resources E technical services, núm. 28, 1984, pp. 4-14.

Stoddart, D. R. (1967), "Growth and structures of Geography", en Transactions of Institute of British Geographers, vol. 41, 1967, pp. 119. 
Sutter, M. y M. Kocher, "Tools for evaluating research output: are citation-based rankings of economics journals stable?", en Evaluation review. vol. 25, 2001. pp. 555-566.

Urbizagástegui Alvarado, Rubén, "Crecimiento de la literatura sobre plantas medicinales del Perú”, en Revista AIBDA, vol. 29, núm. 12, 2008, (De, 18 de julio, 2010: http://www.cnpt.embrapa.br/RevistaAIBDA/v29/v29n1d04.pdf).

Urbizagástegui Alvarado, Rubén y Marlene de Oliveira, "A produtividade dos autores na antropologia Brasileira”, en DataGramaZero: revista de ciência da informação, vol. 2, núm. 6, 2001, (de, 23 de enero, 2010: http://www.dgzero.org/dez01/F_I_art.htm).

Ziman, John M., Introducción al estudio de las ciencias: los aspectos $\mathrm{f}$ losóficos y sociales de la ciencias y la tecnología, Barcelona: Ariel, 1986, 236p.

El conocimiento público: un ensayo sobre la dimensión social de las ciencias, México, D.F.: Fondo de Cultura Económica, 1976, 187p. 


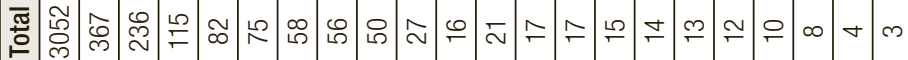
흠

을

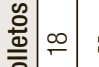

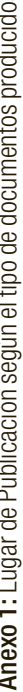

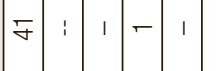

\begin{tabular}{llllllllllllllllllllllllllllllll}
\hline & 1 & 1 & 1 & 1 & - & 1 & 1 & 1 & 1 & 1 & 1 & 1 & 1 & 1 & 1 & 1 & 1 & 1 & 1 & 1
\end{tabular} ๕્)

के

更

む్

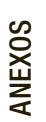

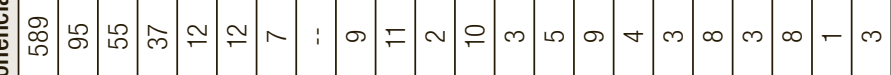

\begin{tabular}{|c} 
을 \\
흥 \\
흔 \\
\hline
\end{tabular}

$\dddot{\mathfrak{c}}$

岗

旁导 こ

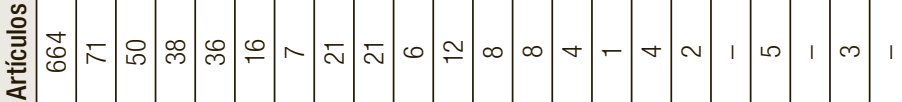

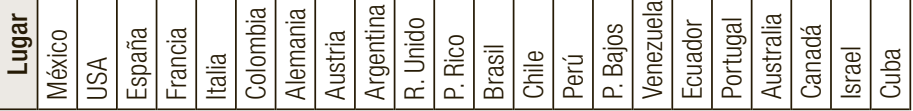




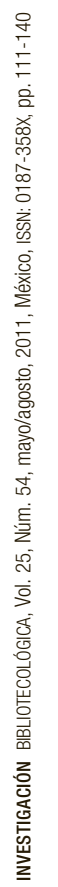

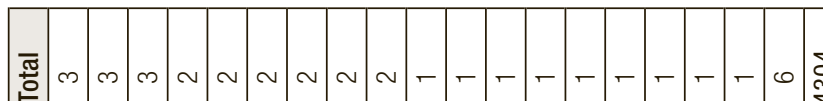

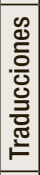

:

읗

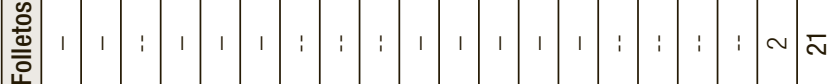

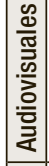

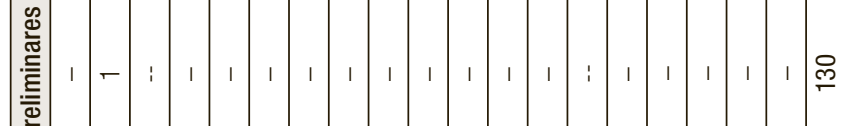

os

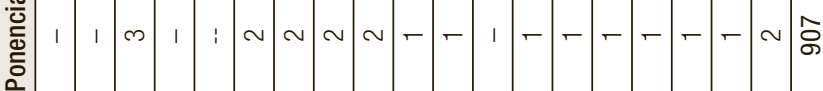

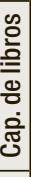

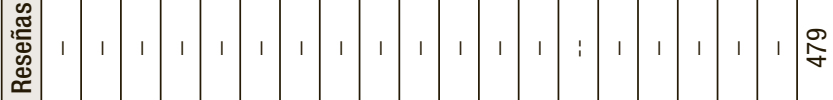

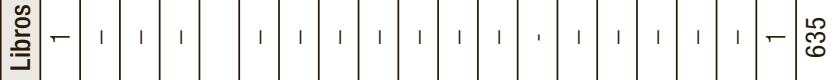

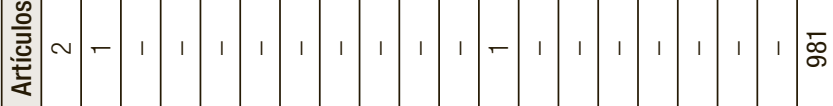

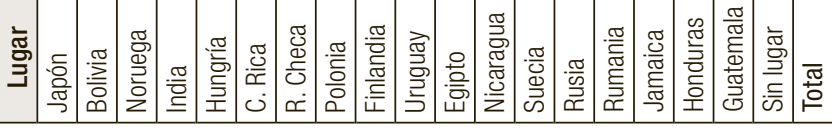




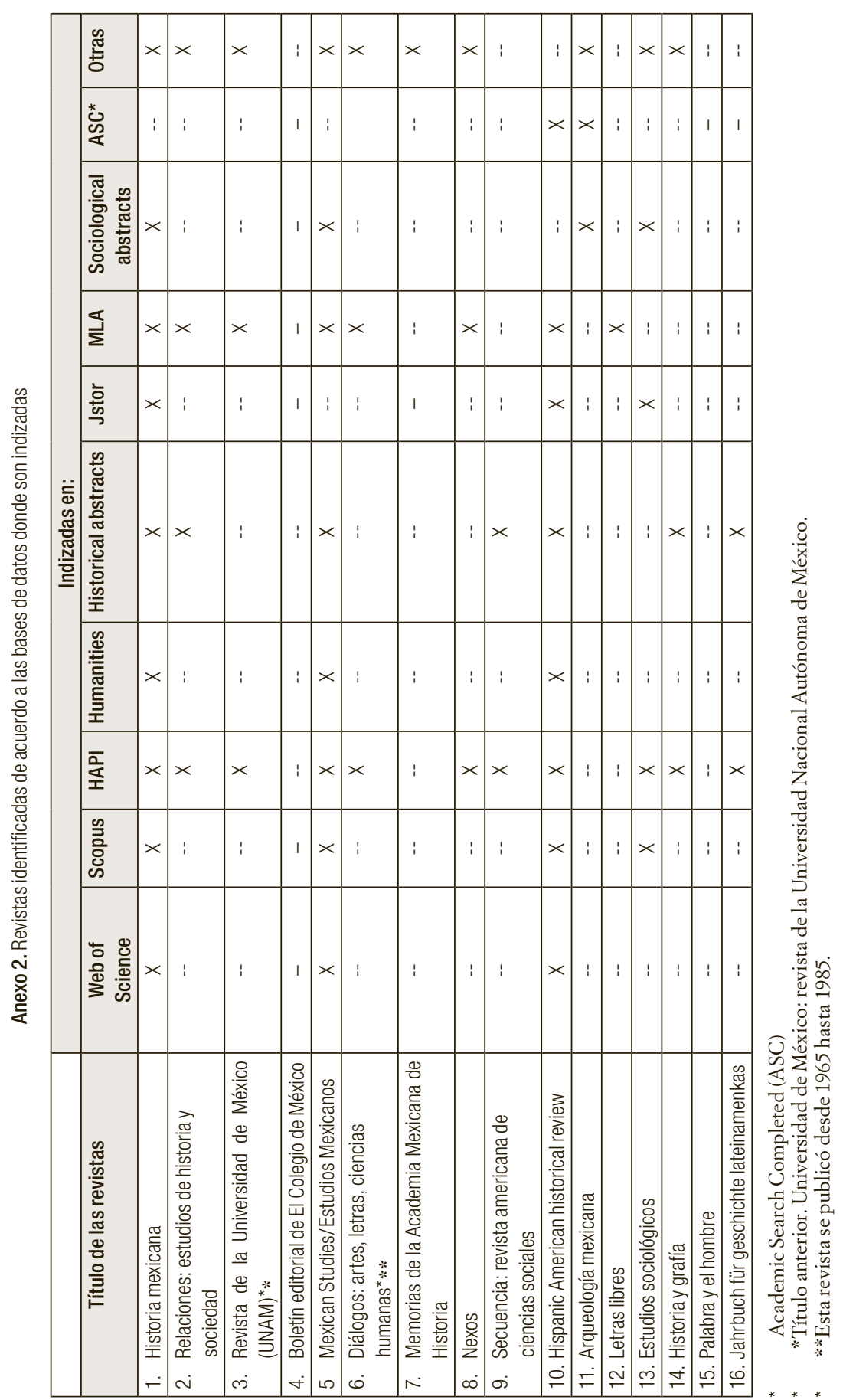




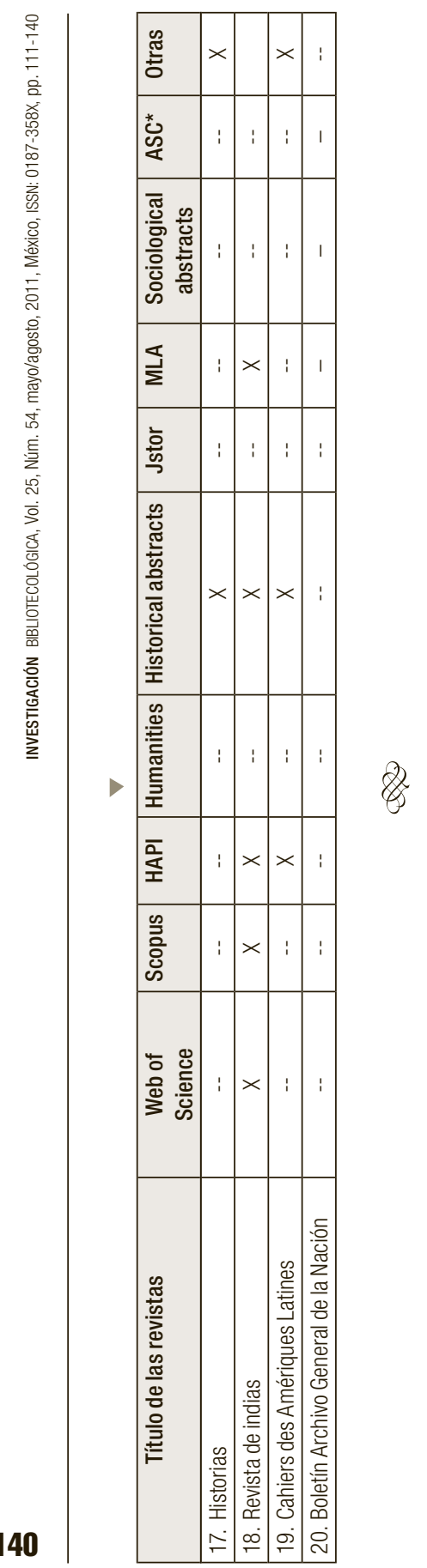

